\title{
Relation \& Association of Mean Ocular Perfusion Pressure in Primary Open Angle Glaucoma
}

\author{
Dr. Anwesha Manna ${ }^{1}$, Dr.Vishalakshi Subramanium ${ }^{1}$, Dr.Sudha Jeyaram ${ }^{1}$

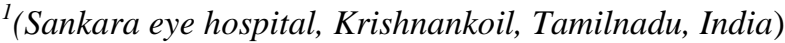

\begin{abstract}
Purpose: To find out relationship of mean ocular perfusion pressure(MOPP), Systolic ocular perfusion pressure $(S P P) \&$ diastolic ocular perfusion pressure with occurrence of primary open angle glaucoma(POAG)

Study Design: Hospital based cross sectional study

Study period: August '2016- February '2017. A period of 6 months.

Setting: Sankara eye hospital, krishnankoil, tamilnadu.

Method: In a period of 6 months we examined total 836 patients of 40-80 years age group from southern part of Tamilnadu, of which 431 patients were male \& 405 were female. All patients are evaluated with dilated fundus bio microscopy with $+90 D$ lens, IOP measurement by applanation tonometer, gonioscopy by goldman 4 mirror goniolens\& Humphrey 30-2 perimetry test. Only primary open angle glaucoma patients were included. Blood pressure was checked in each patient with digital BP measuring instrument. After collecting all data MOPP, $S P P, D P P$ were calculated.

Result: The mean IOP in POAG group was $21.8 \pm 8.4 \mathrm{~mm}$ of $\mathrm{Hg}$, whereas in normal group it was $15.7 \pm 4.2 \mathrm{~mm}$ of $\mathrm{Hg} . \mathrm{MOPP}$ in POAG patients was $59.9 \pm 9.4 \mathrm{~mm}$ of $\mathrm{Hg}$ \& normal group $62.7 \pm 10.4 \mathrm{~mm}$ of Hg. SPP \& DPP in POAG patients were $136.2 \pm 15.4$ \& $62.2 \pm 11.8 \mathrm{~mm}$ of $\mathrm{Hg}$. In normal group SPP \& DPP was $134.6 \pm 18.4 \mathrm{~mm}$ of $\mathrm{Hg} \& 64.6 \pm 10.6 \mathrm{~mm}$ of $\mathrm{Hg}$ respectively. The relation of $O P P$ in POAG was done by multivariate logistic regression analysis, where pressure related factors i.e. MOPP, SPP, DPP were taken as variable. Lower DBP, lower MOPP was significantly associated with OAG risk. Lower DBP (OR= 1.65; 95\% CI, $1.12-2.82$, comparing persons in the lowest quartile with those in highest quartile [Q1 vs. Q4] of DBP), lower MOPP $(O R=1.63 ; 95 \%$ CI, 1.02-2.85; [Q1 vs. Q4]) and lower DPP $(O R,=1.56$; 95\% CI, 1.23-2.92; [Q1 vs. Q4]) were significantly associated with OAG risk. Higher SBP wasnot associated with increased risk of glaucoma( $p=$ 0.64)
\end{abstract}

Keywords: $P O A G, M O P P$, ocular perfusion pressure, SPP, DPP

\section{Introduction}

Glaucoma is among the most common causes of irreversible vision loss worldwide. While its primary etiology remains incompletely understood, we have developed a robust understanding of the risk factors that contribute to the development and progression of the disease. Among these, intraocular pressure (IOP) remains the most important risk factor, both because of its strength of association with the disease and because it remains the only modifiable risk factor. In addition to IOP, vascular factors have long been suspected of playing a role in the glaucomatous process. Evidence from epidemiologic studies and clinical trials alike suggests that ocular perfusion pressure (OPP) - in simplest terms, the difference between IOP and systemic blood pressure (BP) - as well as other factors such as BP, vasospasm, and ischemia may all contribute to glaucoma risk. ${ }^{1-3}$

A multitude of recent literature suggest that, apart from mechanical effects of increased intraocular pressure (IOP), ONH vascular insufficiency plays an important role in the pathogenesis of glaucomatous optic neuropathy. The Baltimore Eye Survey revealed that around $50 \%$ of patients with primary open-angle glaucoma (POAG) had an initial IOP of $<21 \mathrm{~mm} \mathrm{Hg}$ at the time of diagnosis, with around $20 \%$ having normal IOP for all first 3 visits. Asians show a particular tendency for this subtype. In Korean- and Japanese-based cross-sectional epidemiological studies, $94.4 \%$ and $92 \%$ had IOP of $<21 \mathrm{~mm} \mathrm{Hg}$, respectively. ${ }^{6}$

Ocular perfusion pressure can be thought of as the pressure at which blood enters the eye. Dr Liebmann. ${ }^{3}$

Mean OPP (MPP) is the difference between mean arterial BP and IOP. Mean arterial BP is calculated using a 1 formula that accounts for diastole taking up most of the cardiac cycle. Mean ocular perfusion pressure (MOPP) $=2 / 3($ mean arterial pressure $-\mathrm{IOP})$, where mean arterial pressure $(\mathrm{MAP})=\mathrm{DBP}+1 / 3(\mathrm{SBP}-\mathrm{DBP})$, systolic perfusion pressure $(\mathrm{SPP})=\mathrm{SBP}-\mathrm{IOP}$, and diastolic perfusion pressure $(\mathrm{DPP})=\mathrm{DBP}-\mathrm{IOP}$.

Evidence has progressively accumulated that there is vascular insufficiency in the $\mathrm{ONH}$ in both anterior ischemic optic neuropathy (AION) and glaucomatous optic neuropathy (GON)-two major causes of blindness or of seriously impaired vision in man. ${ }^{1}$

A vascular mechanism has been postulated in the pathogenesis of glaucomatous optic nerve damage; specifically, an inadequate or unstable ocular blood supply causes ischemic damage and/or reperfusion injury to 
the optic nerve tissue and axons. This vascular concept is support by the reported associations of glaucomatous optic neuropathy with arterial hypertension, arterial hypotension, cardiovascular disease, migraines, vasospasm, and other circulation disorders. ${ }^{2}$

Lower ocular perfusion pressure is a risk factor OPP=BP-IOP. Blood pressure is related to IOP.It is not clear whether low BP is a risk factor of glaucoma or not in individual patient. ${ }^{4}$

Aim

To find out relationship of mean ocular perfusion pressure(MOPP), Systolic ocular perfusion pressure (SPP) \& diastolic perfusion pressure with occurrence of primary open angle glaucoma(POAG).

Type of study : Population based cross sectional study.

Place of study: Sankara eye hospital, krishnankoil, tamilnadu, India.

Selection of patient:

Inclusion Criteria : 1) Age more than 40 years both male \& female

2) patients with primary open angle glaucoma, diagnosed after applanation tonometry, fundus \& cup-disc ratio evaluation, gonioscopy, $30-2$ perimetry by HFA.

Exclusion Criteria: 1) Patients of angle closure glaucoma, juvenile glaucoma, all types of secondary glaucoma

\section{Method \& Material}

During 2016 August to 2017 February, we examined 836 patients persons of Sothern part of Tamilnadu aged 40 to 80 years. Visual acuity and refraction were performed by experienced optometrists. A comprehensive ocular examination including slit lamp biomicroscopy, applanation tonometry, gonioscopy, indirect ophthalmoscopy as well as stereo-biomicroscopy of the disc and macula (using a non-contact $+90 \mathrm{D}$ lens) was performed in a standard manner after dilatation of pupil by one of three ophthalmologists participating in the study. Gonioscopy was performed in a routine manner using the Goldmann 4 mirror lens.

An automated visual field (Humphrey Field Analyzer 24-2 SITA FAST program) was scheduled on the following indications: intraocular pressure (IOP) $>21 \mathrm{mmHg}, \mathrm{CDR} \geq 0.6$ in either eye, difference in CDR $>0.2$ between the two eyes and /or the presence of glaucomatous features. Unreliable fields were repeated during another session. If an advanced field defect precluded a successful 24-2 examination, the macular program or a 10-2 program was used.

For the purpose of this study, primary open angle glaucoma (POAG) was defined as the presence of elevated IOP and /or glaucomatous disc changes in the presence of typical glaucomatous field defects, an open angle on gonioscopy and no evidence of a secondary cause. We selected 67 patients with such criteria.

\section{Definition of glaucoma:}

Patients with suspected glaucoma all underwent visual field testing and were defined by any of the following criteria: (1) IOP greater then $21 \mathrm{~mm} \mathrm{Hg}$; (2) VCDR > 0.6 or VCDR asymmetry > 0.2; (3) abnormal anterior segment deposit consistent with pseudoexfoliation or pigment dispersion syndrome; (4) occludable angle, defined as posterior trabecular meshwork seen for $180^{\circ}$ or less of the angle circumference during static gonioscopy; (5) peripheral anterior synechiae or other findings consistent with secondary glaucoma; and (6) known history of glaucoma. Only Open angle cases were included in this study.
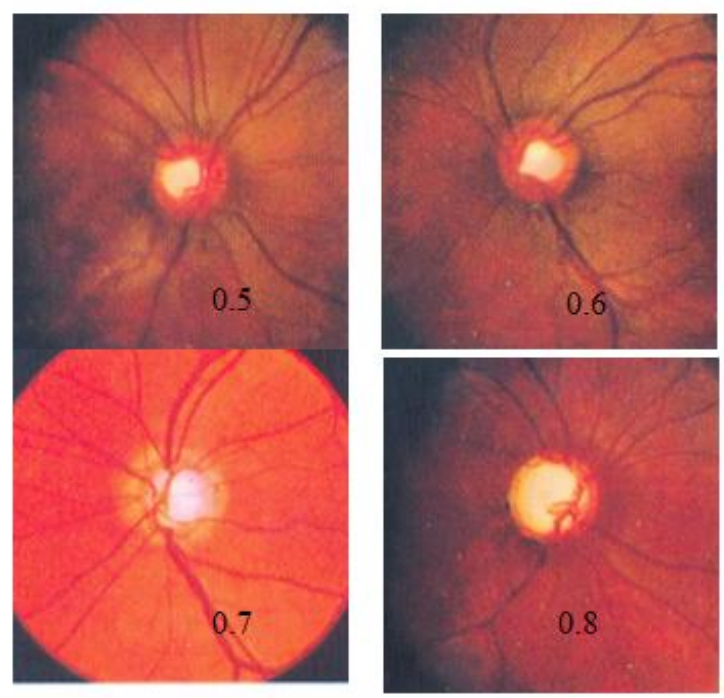
Glaucoma cases were classified into three categories, according to ISGEO ( International Society of geographical \& Epidemiological Ophthalmology) criteria: category 1, optic disc abnormality (VCDR/VCDR asymmetry $>97.5$ th percentile or neuroretinal rim width between 11 and 1 o'clock or 5 and 7 o'clock $>0.1$ VCDR) with a corresponding glaucomatous visual field defect; category 2, a severely damaged optic disc (VCDR or VCDR asymmetry $>99.5$ th percentile) in the absence of an adequate visual field test; category 3 , no visual field or optic disc data, blindness (corrected visual acuity, $<3 / 60$ ), and prior glaucoma surgery IOP $>99.5$ th percentile. For cases classified as category 1 or 2 , there could be no other explanation for the VCDR finding (e.g., dysplastic disc or marked anisometropia) or visual field defect (e.g., branch retinal vein occlusion, macular degeneration, or cerebrovascular disease). ${ }^{5}$

\begin{tabular}{|l|}
\hline The diagnosis of glaucoma in cross sectional prevalence surveys (The diagnosis is made \\
according to three levels of evidence) \\
\hline Category 1 diagnosis (structural and functional evidence) \\
Eyes with a CDR or CDR asymmetry $>97.5$ th percentile for the \\
normal population, or a neuroretinal rim width reduced to $<0.1$ CDR \\
(between 11 to 1 o'clock or 5 to 7 o'clock) that also showed a \\
definite visual field defect consistent with glaucoma. \\
\hline Category 2 diagnosis (advanced structural damage with unproved \\
field loss) \\
If the subject could not satisfactorily complete visual field testing but \\
had a CDR or CDR asymmetry $>99.5$ th percentile for the normal \\
population, glaucoma was diagnosed solely on the structural \\
evidence. \\
In diagnosing category 1 or 2 glaucoma, there should be no \\
alternative explanation for CDR findings (dysplastic disc or marked \\
anisometropia) or the visual field defect (retinal vascular disease, \\
macular degeneration, or cerebrovascular disease). \\
\hline Category 3 diagnosis (Optic disc not seen. Field test impossible) \\
If it is not possible to examine the optic disc, glaucoma is diagnosed if: \\
(A) The visual acuity <3/60 and the IOP $>99.5$ th percentile, or \\
(B) The visual acuity $<3 / 60$ and the eye shows evidence of glaucoma \\
filtering surgery, or medical records were available confirming \\
glaucomatous visual morbidity.
\end{tabular}

\section{Calculation of MOPP(mean ocular perfusion Pressure):}

OPP or ocular perfusion pressure is defined as mean, systolic or diastolic OPP. Mean OPP (MOPP) can be calculated as $2 / 3$ of the mean arterial $\mathrm{BP}-\mathrm{IOP}$; where mean arterial pressure $=$ diastolic $\mathrm{BP}+1 / 3$ (systolic $\mathrm{BP}$ - diastolic BP). The factor $2 / 3$ accounts for the drop in blood pressure between the brachial and ophthalmic artery when the subject is seated (Riva et al. 1986) and the fact that the orbital arteries are further downstream. ${ }^{7}$ Systolic ocular perfusion pressure (SOPP) is defined as the difference between systemic systolic BP and IOP, whereas diastolic ocular perfusion pressure (DOPP) equals systemic diastolic BP - IOP. DOPP is especially useful in displaying the lowest OPP values. In our study BP was measured by automatic digital BP machine, with the patient being in supine position \& after 1 hour of rest. In each patient along with SBP \& DBP, MOPP, SOPP,DOPP were calculated.

\section{Result \& Analysis}

In this study we have evaluated total 836 patients, of which 67 mate the criteria of primary open angle glaucoma. Male patients were $34 \&$ female patients were 33 . The following bar \& pie diagram shows total number of normal patients \& glaucoma patients in different age groups.

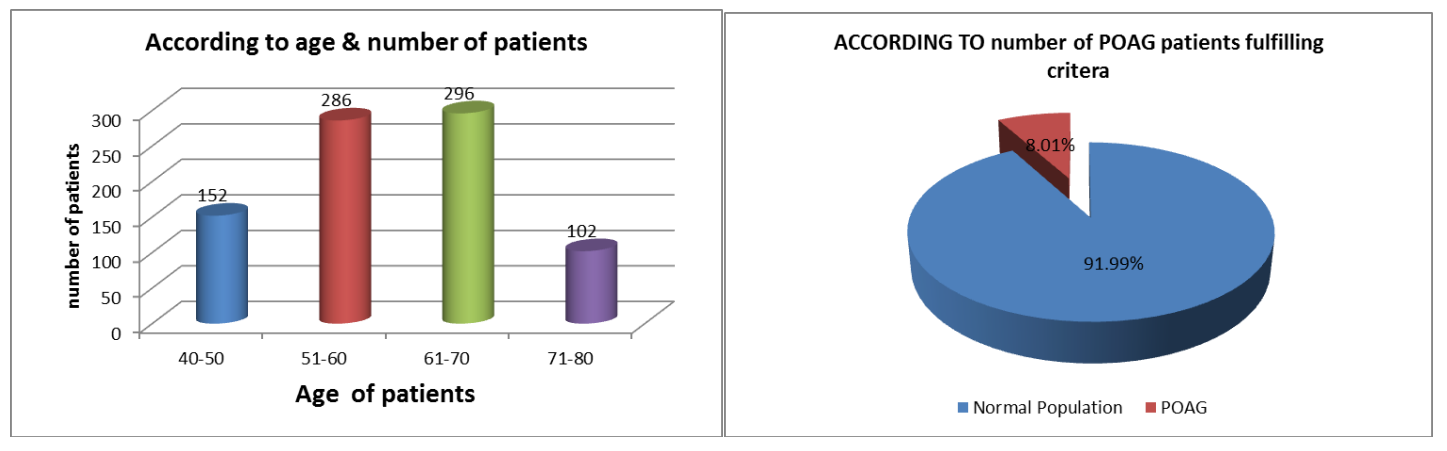




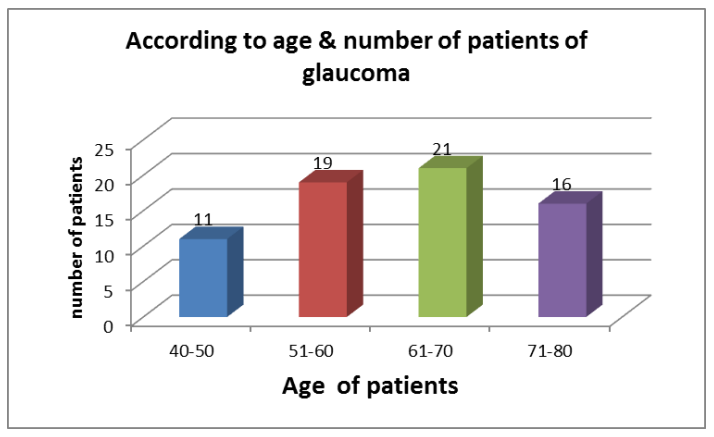

After measuring systolic \& diastolic BP \& IOP by applanation tonometer calculation done for MOPP, SPP, DPP. Following diagram shows the mean value in normal \& POAG groups. The mean IOP in POAG group was $21.8 \pm 8.4 \mathrm{~mm}$ of $\mathrm{Hg}$, whereas in normal group it was $15.7 \pm 4.2 \mathrm{~mm}$ of $\mathrm{Hg}$. Systolic BP \& diastolic BP in POAG patients were respectively $150.8 \pm 10.2 \& 78.2 \pm 9.6 \mathrm{~mm}$ of $\mathrm{Hg}$. In normal population it was 147.8 $\pm 11.3 \& 80.6 \pm 12.7 \mathrm{~mm}$ of $\mathrm{Hg}$ respectively. After calculation MOPP in POAG patients was $59.9 \pm 9.4 \mathrm{~mm}$ of Hg\& normal group $62.7 \pm 10.4 \mathrm{~mm}$ of $\mathrm{Hg}$. SPP/SOPP \& DPP/DOPP (systolic ocular perfusion pressure \& diastolic ocular perfusion pressure) in POAG patients were $136.2 \pm 15.4 \& 62.2 \pm 11.8 \mathrm{~mm}$ of $\mathrm{Hg}$. In normal group SOPP \& DOPP was $134.6 \pm 18.4 \mathrm{~mm}$ of $\mathrm{Hg} \& 64.6 \pm 10.6 \mathrm{~mm}$ of $\mathrm{Hg}$ respectively.

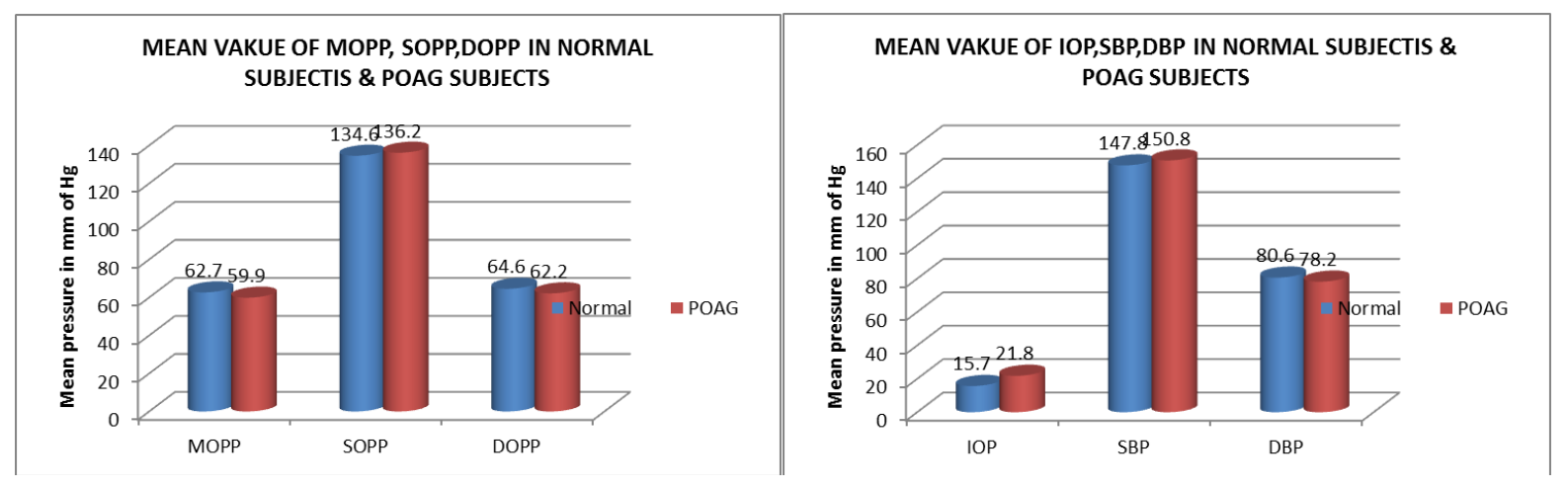

Analysis of result:

The relation of OPP in POAG was done by multivariate logistic regression analysis, where pressure related factors i.e. MOPP,SPP,DPP were taken as variable. $\mathrm{P}$ value of $\iota 0.05 \&$ OR of 1.00 was taken to find out association.Lower DBP , lower MOPP were significantly associated with OAG risk. lower DBP (OR=1.65; 95\% CI, $1.12-2.82$, comparing persons in the lowest quartile with those in highest quartile [Q1 vs. Q4] of DBP), lower MOPP (OR=1.63; 95\% CI, 1.02-2.85; [Q1 vs. Q4]) and lower DPP (OR, $=1.56$; 95\% CI, $1.23-$ 2.92; [Q1 vs. Q4]) were significantly associated with OAG risk. Higher SBP wasnot associated with increased risk of glaucoma $(\mathrm{p}=0.64) \mathrm{MOPP}$ and SPP increased with age (both $\mathrm{P}<0.005)$, whereas DPP was not related to age $(\mathrm{P}=0.7)$ When compared pressure in men \& women, men group had higher MOPP and higher DOPP, but lower SOPP, than did women ( $\mathrm{p}=.012 \& \mathrm{p}=0.09$ respectively) in t-test. Both were statistically significant.

\section{Discussion}

POAG is a disease of optic nerve with multiple aetiology. IOP is the only modifiable factor. We conducted this cross sectional study to find out association in southern part of Tamilnadu district, India.In this cross sectional study we found mean SBP \& DBP was $147.8 \pm 11.3 \& 80.6 \pm 12.7 \mathrm{~mm}$ of $\mathrm{Hg}$ in normal group. Mean IOP in POAG group was $21.8 \pm 8.4 \mathrm{~mm}$ of $\mathrm{Hg}$, whereas in normal group it was $15.7 \pm 4.2 \mathrm{~mm}$ of $\mathrm{Hg}$. In Beaver Dam Eye Study the result of mean BP \& mean IOP was $146.9 \mathrm{~mm}$ of $\mathrm{Hg} \& 15.4 \mathrm{~mm}$ of $\mathrm{Hg}^{8}{ }^{8}$ The results in this study is comparable in other studies like Singapore malaye eye study ${ }^{2}$. But in Barbados Eye Study the mean IOP is little higher, $18 \mathrm{~mm}$ of $\mathrm{Hg}^{9}{ }^{9}$

Blue Mountains Eye Studyby Mitchell P et al found that a higher SPP was associated with OAG risk $(\mathrm{OR}, 1.09 ; \mathrm{P}=0.05)$ \&a higher DPP was associated with reduced risk of ocular hypertension $(\mathrm{OR}, 0.78 ; \mathrm{P}=$ $0.0008) .{ }^{10}$ But in Singapore Maley eye study SBP was not associated with increased risk of POAG. ${ }^{2}$ In our study we found a positive association of lower DBP with POAG.The Egna-Neumarkt Study has shown a positive correlation between systemic blood pressure (SBP) and IOP, and an association was found between diagnosis of primary open-angle glaucoma and systemic hypertension. Lower DPPwas associated with a marked, progressive increase in the frequency of glaucoma in that study ${ }^{11}$. But our study although low DBP was associated with 
glaucoma, SBP was not positively associated.Hulsman CA et al in their study had mentioned low diastolic perfusion pressure $(<50 \mathrm{~mm} \mathrm{Hg})$ was inversely associated with ntOAG (OR, 0.25; 95\% CI, 0.10-0.63) and positively associated with htOAG (OR, 4.68; 95\% CI, 1.29-17.01. They had found htOAG (hypertensive open angle glaucoma) was associated with high pulse pressure and, only in persons treated for systemic hypertension, with low diastolic perfusion pressure. ${ }^{12}$

M. Cristina Leske et al in review also has mentioned that there is positive association exists between $\mathrm{BP}$ and IOP \& low ocular perfusion pressure is significantly related to OAG. ${ }^{13}$ The difference in result in few findings may be due to calculation \& measurement of BP \& IOP. We have taken a single finding for both BP \& IOP. A measurement of 24-hour MOPP or DPP may provide more precise information for assessing the relationship of perfusion pressure and risk of OAG. Another thing is that, the circadian changes we have not considered.

\section{Conclusion}

Till date different studies are done by research groups \& a positive association has been found by different researchers between IOP \& ocular perfusion pressure. In our study we found that a positive significant association persists with low MOPP, SPP, DPP. Low DBP is also significantly associated, but SBP is not significantly associated. So,along with high IOP a vascular mechanism is there behind the occurrence of POAG.

\section{References}

[1]. Hayreh SS. Blood flow in the optic nerve head and factors that may influence it. Prog Retin Eye Res. 2001;20:595-624.

[2]. Distribution of Ocular Perfusion Pressure and Its Relationship with Open-Angle Glaucoma: The Singapore Malay Eye Study. Yingfeng Zheng, Tien Y. Wong, Paul Mitchell et al, Investigative_ophthalmology_visual_science. DOI: 10.1167/iovs.09-4867 • Source: PubMed.

[3]. Ocular Perfusion Pressure in Glaucoma, Robert N. Weinreb, MD, David S. Greenfield, MD, November 1, 2015

[4]. Nicolela MT, Walman BE, Buckley AR, Drance SM. Ocular hypertension and primary open-angle glaucoma: a comparative study of their retrobulbar blood flow velocity. J Glaucoma. 1996;5:308-310

[5]. Paul J Foster, Ralf Buhrmann, Harry A Quigley, Gordon J Johnson, The definition and classification of glaucoma in prevalence surveys. 3 August 2001, British journal of ophthalmology.

[6]. Karen Kar-Wun Chan, Jonathan Chun-Ho Ho, Ocular perfusion pressure and glaucoma: a review. HongKong Journal of Ophthalmology Vol.20 issue No.1

[7]. Vital P. Costa, Alon Harris, Douglas Anderson. Ocular perfusion pressure in glaucoma. Acta Ophthalmol. 2014: 92: e252-e266

[8]. Klein BE, Klein R, Linton KL. Intraocular pressure in an American community. The Beaver Dam eye study. Invest Ophthalmol Vis Sci. 1992;33:2224-2228.

[9]. Leske MC, Wu SY, Hennis A, Honkanen R, Nemesure B. Risk factors for incident open-angle glaucoma The Barbados Eye Studies. Ophthalmology. 2008;115:85-93.

[10]. Mitchell P, Lee AJ, Rochtchina E, Wang JJ. Open-angle glaucoma and systemic hypertension: The Blue Mountains Eye Study. J Glaucoma. 2004;13:319.

[11]. Bonomi L, Marchini G, Marraffa M, Bernardi P, Morbio R, Varotto A. Vascular risk factors for primary open angle glaucoma the Egna-Neumarkt Study. Ophthalmology. 2000;107:1287-1293.

[12]. Hulsman CA, Vingerling JR, Hofman A, Witteman JC, de Jong PT. Blood pressure, arterial stiffness, and open-angle glaucoma: the Rotterdam study. Arch Ophthalmol. 2007;125:805-812.

[13]. Leske MC. Ocular perfusion pressure and glaucoma: clinical trial and epidemiologic findings. Curr Opin Ophthalmol. 2009;20:7378 . 